Fanum

Sociológico
Forum Sociológico

Série II

17 | 2007

Envelhecimento activo. Um novo paradigma

\title{
Envelhecimento activo : uma via para o bem-estar
}

\author{
Lídia Maria Pereira Lopes
}

\section{OpenEdition}

Journals

Edição electrónica

URL: https://journals.openedition.org/sociologico/1639

DOI: 10.4000/sociologico.1639

ISSN: 2182-7427

Editora

CICS.NOVA - Centro Interdisciplinar de Ciências Sociais da Universidade Nova de Lisboa

Edição impressa

Data de publição: 1 janeiro 2007

Paginação: 65-68

ISSN: 0872-8380

\section{Refêrencia eletrónica}

Lídia Maria Pereira Lopes, «Envelhecimento activo : uma via para o bem-estar», Forum Sociológico [Online], 17 | 2007, posto online no dia 01 janeiro 2007, consultado o 29 março 2022. URL: http:// journals.openedition.org/sociologico/1639; DOI: https://doi.org/10.4000/sociologico.1639

Este documento foi criado de forma automática no dia 29 março 2022.

(c) CICS.NOVA 


\title{
Envelhecimento activo : uma via para o bem-estar
}

\author{
Lídia Maria Pereira Lopes
}

\section{Introdução}

1 Nos últimos tempos temos sido confrontados com uma inquietação crescente devido ao envelhecimento da população, tanto a nível nacional como mundial. Nos países mais desenvolvidos o aumento do tempo de vida ultrapassa cenários e expectativas anteriormente definidas. Este fenómeno deve-se essencialmente ao desenvolvimento económico e social das populações, e ainda a uma melhoria do acesso aos cuidados de saúde, à evolução do conhecimento médico, assim como de técnicas de diagnostico e do próprio desenvolvimento farmacológico (Fernandes, 2007).

2 Em Portugal, o número de idosos tem também aumentado, sobretudo o grupo de $80 \mathrm{e}$ mais anos, tendo-se registado um aumento de $35 \%$ entre os anos de 1990 e 2006. Esta população representava 4,1 \% da população total em 2006 (INE, 2007).

3 Sabe-se que os últimos anos de vida são muitas vezes acompanhados por um aumento das situações de doença e de incapacidade (Portugal, 2006). As necessidades apresentadas situam-se entre a protecção social e a saúde.

o Plano Nacional para a Saúde das Pessoas Idosas, elaborado pela Direcção Geral de Saúde afirma que as políticas e estratégias implementadas raramente se baseiam na promoção de saúde e dificilmente se envolve os idosos no seu planeamento e avaliação. Mas a promoção de saúde e os cuidados de prevenção, dirigidos às pessoas idosas, aumentam a longevidade, melhoram a saúde e a qualidade de vida, e ajudam a racionalizar os recursos da sociedade (Portugal, 2006). Fonseca afirma que a possibilidade de envelhecer com sucesso, numa perspectiva global, valorizando os seus aspectos psicológicos e sociais, "não cai do céu, é preciso desejá-lo, planeá-lo, fazer esforço para o alcançar" (Fonseca, 2005 : 281). Envelhecer é cada vez mais um desafio para quem envelhece e para quem apoia o envelhecimento (Lopes, 2007). É necessário por isso reflectir, debater e compreender para assim encontrar estratégias que ajudem a 
superar este novo desafio com êxito. É importante olhar os mais velhos como fonte de resolução das questões que se colocam.

\section{Envelhecimento activo}

5 A Organização Mundial da Saúde, no âmbito da II Assembleia Mundial sobre Envelhecimento promovida pelas Nações Unidas, lançou o conceito de envelhecimento activo como pilar para a sustentabilidade da problemática que o aumento dos idosos trouxe à sociedade. E define este conceito como "o processo que consiste em optimizar as possibilidades de boa saúde, de participação e de segurança a fim de aumentar a qualidade de vida durante a velhice" (OMS, 2002 : 12). Uma boa saúde torna-se assim uma condição essencial para que as pessoas idosas possam manter-se independentes e continuar a viver com as suas famílias, na sua comunidade. Para que o conceito de "envelhecimento activo" se torne operacional, este deve estar enraizado solidamente na realidade, nomeadamente na construção de actividades de promoção de saúde e no acesso universal das pessoas de idade aos serviços de saúde ao longo da vida (NU, 2002). Mas "activo" não se refere apenas à capacidade de estar fisicamente enérgico, refere-se sim ao envolvimento contínuo dos idosos nas questões sociais, económicas, espirituais, culturais e cívicas (Vallespir, Morey, 2007).

6 A Organização Mundial de Saúde realça a necessidade de pôr em prática políticas e programas que ajudem os idosos a envelhecer mantendo-se activos, isto é que favoreçam a saúde, a participação e a segurança destes cidadãos. Estas políticas e programas devem apoiar-se nos direitos, nas preferências e nas capacidades das pessoas idosas, e não apenas nas necessidades que apresentam (OMS, 2002). É imprescindível assim que os sistemas de saúde concentrem os esforços na promoção da saúde e na prevenção das enfermidades. Neste sentido os serviços de saúde e sociais deverão trabalhar em conjunto. Um dos desafios das políticas de saúde é de encontrar o equilíbrio entre o cuidar de si próprio, o apoio informal e os cuidados prestados por profissionais (Vallespir e Morey, 2007). Cada pessoa, cada família deve prever e preparar-se para o envelhecimento e fazer esforços pessoais para adoptar práticas favoráveis à saúde ao longo da vida (OMS, 2002).

7 No Plano Nacional de Saúde 2004-2010 é dada particular atenção ao "envelhecimento activo", no qual se propõem algumas orientações estratégicas e intervenções necessárias para uma reorganização dos cuidados aos idosos, numa perspectiva integrada, com uma abordagem multidisciplinar e intervenção inter-sectorial. As principais estratégias são: adequar os cuidados de saúde às necessidades específicas dos idosos ; actuar sobre determinantes de autonomia e independência ; e promover e desenvolver, intersectorialmente, ambientes capacitadores de autonomia e independência dos idosos (Portugal, 2004).

\section{Políticas e estratégias baseadas no envelhecimento activo}

8 A Organização Mundial de Saúde lança o debate de se encontrar uma resposta integrada dos sistemas de cuidados de saúde, perante o envelhecimento rápido da população. Refere mesmo, a necessidade de se criar uma base de conhecimento destinada a ajudar 
os países a reorientar as políticas para os sistemas de cuidados de saúde e de protecção social integrados, ao serviço dos mais idosos (OMS, 2005).

o Conselho Económico e Social das Nações Unidas afirma também que a instauração de serviços de cuidados de saúde e sociais acessíveis e de boa qualidade, sobretudo os serviços de saúde de longa duração, é uma das dificuldades mais agudizantes para as sociedades que envelhecem com rapidez. Igualmente importante é procurar que haja uma colaboração estreita entre os profissionais dos serviços de assistência médica e os serviços de assistência social que atendem as pessoas de idade (NU, 2007). Em Portugal, as orientações actuais da política nesta área têm-se centrado nos cuidados integrados e articulados entre segurança social e a saúde, com o Programa de Cuidados Continuados e de Apoio Integrado. Espera-se que este programa abranja todos os idosos em situação de fragilidade, e que se inicie uma cultura de estreita colaboração entre o sector da saúde e o sector social.

10 Foi elaborado um relatório no Reino Unido que procura inverter a dinâmica dos cuidados prestados aos idosos. Actualmente, os serviços para idosos estão, ainda, concentrados nas respostas aos cuidados agudos e aos idosos frágeis. Os serviços futuros necessitam de alterar esta visão, encontrar estratégias de promoção e de bemestar para as pessoas idosas e encorajar estilos de vida saudáveis para os idosos. Os idosos e os cuidadores informais devem estar no centro interactivo com os serviços universais numa comunidade activa. Os objectivos deverão ser definidos com empowerment através da participação de todos, nomeadamente os idosos, as famílias e as instituições envolvidas. Os serviços especializados, como os hospitais, estarão na periferia desta dinâmica e só interagem quando houver necessidade. A nível local, é importante clarificar responsabilidades, para que a estratégia de desenvolvimento e coordenação dos recursos seja vital. O futuro está em equipas multidisciplinares, interagindo com base nas comunidades locais e promovendo independência e autonomia. Aumentam-se as expectativas do bem-estar, direitos, escolhas e protecção. É possível a promoção de um envelhecimento bem sucedido para todos, incluindo os muito velhos, mesmo em situação de diminuição de independência. Estas estratégias têm por base o livre acesso a alojamentos protegidos para pessoas idosas muito frágeis e com perda de autonomia, o bom acesso aos cuidados de saúde, o incentivo à formação ao longo da vida, a criação de outros serviços que sustentem a interacção das comunidades, construindo comunidades seguras. No futuro, os serviços no futuro necessitam de ser conduzidos, responsabilizados na sociedade, integrados, baseados na comunidade, flexíveis e facilmente acessíveis. $O$ que levaria à redução de lares para idosos com respostas mínimas às suas necessidades e ao alargamento de serviços comunitários. O desafio é melhorar o serviço social e expandir as respostas à população. O objectivo é conceber num mesmo espaço os serviços de saúde e sociais, abertos 24 horas, com orçamentos conjuntos e construir residências protegidas sem fronteiras com a comunidade (Association of Directors of Social Services, 2003).

11 Graças a este novo conhecimento, as sociedades estarão bem mais preparadas para prestar serviços de saúde e apoio social e para enfrentar o aumento da idade média da população. A crescente sensibilidade para com o envelhecimento é um claro indício do avanço neste âmbito (Âgren e Berensson, 2006). o Comité Económico e Social da União Europeia refere que a ordem pela qual os cuidados foram construídos ao longo dos últimos anos, em que a estrutura demográfica e as necessidades eram diferentes, devem ser repensadas perante a rigidez e a resistência à mudança que caracteriza as 
estruturas organizacionais, as funções dos profissionais e as atitudes culturais. Actualmente, a política de cuidados às pessoas idosas deve orientar-se para a promoção da autonomia e independência pessoal, a partir da integração dos cuidados nas áreas social e de saúde. Pois existem situações onde os limites são fluídos entre os cuidados de saúde e de assistência social. Uma política de saúde eficiente implica repensar as interacções entre estes dois tipos de cuidados, afim de identificar as estruturas, os procedimentos e as tipologias de prestações profissionais mais adaptadas aos contextos actual e futuro (Braghin, 2004).

Se o envelhecimento da população é uma ambição natural de qualquer sociedade, é necessário continuar a desenvolver esforços para proteger a vida dos mais velhos, tornando-se um desafio que se coloca a toda a sociedade. Assim, é importante oferecer condições adequadas aos idosos para viverem com bem-estar, autonomia, integrados na sociedade, com garantia dos meios de subsistência, apoios e cuidados necessários.

\section{Recursos de "bem-estar"}

13 Para Fonseca (2005) há diferenças significativas quanto à forma como o processo de envelhecimento decorre. Por um lado, a importância do contexto cultural de referência, onde a velhice tanto pode ser sinónimo de prestígio social como de doença e de dependência ; por outro, as diferenças de pessoa para pessoa, relativamente a vários aspectos vistos como determinantes para o seu bem-estar, como sejam as condições económicas, a saúde física, as redes sociais de pertença e de apoio. Mas a maioria das pessoas adapta-se às mudanças com a idade e continua a ser autónoma até mesmo quando já tem uma idade muito avançada. A sua capacidade de adaptação não tem que decair com o avanço da idade, havendo todo um conjunto de recursos internos e externos que se afirmam como "recursos de bem-estar", tanto do ponto de vista físico, como psicológico e social, e que ajudam a envelhecer bem (Manciaux, 2003 cit. in Fonseca, 2005). Assim, o desafio do envelhecimento com qualidade irá depender cada vez mais dos suportes sociais. As respostas sociais devem ser organizadas tendo em conta as autoridades locais e a sociedade civil, incluindo as organizações não governamentais, o sector privado, os voluntários, as próprias pessoas idosas, as organizações representantes das pessoas idosas e as que prestam serviços a estas, assim como as famílias e as comunidades (Martín, Gonçalves et al, 2007).

Valleespir e Morey (2007) destacam que segundo vários estudos, os idosos se sentem mais satisfeitos se puderem viver no seio familiar e rodeados pela comunidade onde passaram a maior parte da vida. Os idosos dependentes manifestaram também preferir os cuidados domiciliários aos cuidados institucionalizados. A existência de redes de apoio informais, de relações sociais significativas, é essencial para assegurar a autonomia. Num estudo sobre qualidade de vida à população idosa no Reino Unido, os aspectos mais valorizados pelos idosos a viverem na comunidade foram: ter boas relações com a família e os amigos; desempenhar papéis sociais, como voluntariado e hobbies ; ter boa saúde e funcionalidade ; viver numa boa casa, numa zona simpática e de boa vizinhança; ter uma visão positiva da vida e manter o controlo e a independência (Bowling, Banister et al, 2003, cit. in Paúl e Fonseca, et al 2005).

Para que se envelheça tendo por base os princípios do envelhecimento activo é importante olhar para a pessoa idosa inserida no seu meio, em que os recursos tanto de 
saúde como sociais devem estar disponíveis para todos, segundo as suas necessidades e desejos.

\section{Conclusão}

O paradigma do envelhecimento activo é a chave para promover qualidade de vida e bem-estar até ao fim. Não deve ser apenas uma intenção, mas uma dinâmica que chegue a todos os idosos. A velhice deve ser encarada como uma etapa plena da vida e não como apenas viver na inquietação de ver os seus dias chegarem ao fim. Com saúde, autonomia e inseridos na sua comunidade, os anos da velhice podem ser vividos como uma experiência renovadora e partilhada com as gerações mais novas. As políticas sociais e de saúde devem ter no seu horizonte o envelhecer como saúde, participação e segurança. As estratégias implementadas podem incluir uma nova cultura de cuidar, em que a família e o próprio idoso sejam parceiros das decisões e dos planos de cuidados. Em situação de fragilidade e dependência, é importante manter o idoso inserido na sua comunidade. Os recursos formais e informais devem interligar-se para que haja uma verdadeira continuidade de cuidados, uma rede de proteç̧ão que possibilite a continuidade de vida com qualidade, mesmo quando se transita entre instituições de saúde e sociais ou familiares. Vivemos cada vez mais numa sociedade global em que é fundamental cultivar as relações sociais e afectivas de modo que se tornem em recursos de bem-estar. Envelhecer num ambiente protector é o desafio de construir uma sociedade para todos, sobretudo para os nossos velhos.

\section{Nota da Redacção}

Lídia Maria Pereira Lopes (lidialopes@ensp.unl.pt). Mestre em Saúde Pública pela ENSP/UNL, Enfermeira.

\section{BIBLIOGRAFIA}

ÂGREN, G. e K. Berenson (2006), Healthy Ageing : A Challenge for Europe, The Swedish National Institute of Public Health, http://www.healthyageing. nu/upload/Rome/Healthy_web.pdf (acedido a 12-9-2007).

ASSOCIATION OF DIRECTORS OF SOCIAL SERVICES (2003), All Our Tomorrows - Inverting the Triangle of Care: A joint discussion document on the future of services for older people, Local Government Association, www.adss.org.uk (acedido a 30-6-2007).

BRAGHIN, M. (2004), Santé et soins des personnes âgées, Comité Économique et Social Européen. AVIS de la section spécialisée, Bruxelles, CESE 999/2004 fin IT-DS/CH/mc, http://eescopinions.eesc.europa.eu/eescopiniondocument. aspx ?language $=p t \&$ docnr $=1447 \& y e a r=2004$ (acedido a 22-6-2007).

FERNANDES, A. A. (2007), "Determinantes da Mortalidade e da Longevidade : Portugal numa Perspectiva Europeia (EU 15, 1991-2001)”, Análise Social, XLII, pp. 419-443. 
FONSECA, A. M. (2005), “O envelhecimento bem-sucedido”, in C. Paul e A. M. Fonseca (coords.), Envelhecer em Portugal, Lisboa, Climepsi Editores, pp. 281-308.

INSTITUTO NACIONAL DE ESTATÍ́STICA (INE) (2007), "Dia Mundial do idoso : 1 de Outubro de 2007”, Destaque, Informação à Comunicação Social, www.ine.pt (acedido 12-1-2008).

LOPES, L. (2007), “Necessidades e Estratégias na Dependência : Uma visão da família”, Revista Portuguesa de Saúde Pública 25 (1), pp. 39-46.

MARTÍN, I. e D. Gonçalves, et al (2007), "Políticas Sociais para a Terceira Idade”, in A.R. Osório e F. C. Pinto (coords.), Pessoas Idosas : Contexto Social e Intervenção Educativa, Lisboa, Instituto Piaget, pp. 131-179.

NACIONES UNIDAS (NU) (2002), Informe de la Segunda Asamblea Mundial sobre el Envejecimiento, Madrid, http://daccessdds.un.org/org/UNDOC/ GEN/N02/397/54/PDF/NO239754.pdf (acedido a 12-5-2005).

NACIONES UNIDAS (NU) (2007), Principales acontecimientos en la esfera del envejecimiento desde la segunda Asamblea Mundial sobre el Envejecimiento, Comisión de Desarrollo Social. Consejo Económico y Social 45 periodo de sesiones, http://daccessdds.un.org/doc/UNDOC/GEN/ N06/625/90/PDF/N0662590.pdf?OpenElement (acedido a 18-6-2007).

ORGANISATION MONDIALE DE LA SANTÉ (OMS) (2002), Vieillir en restant actif : Cadre d'orientation, http://whqlibdoc.who.int/hq/2002/WHO_NMH_NPH_02.8_fre.pdf (acedido a 27-4-2005).

ORGANISATION MONDIALE DE LA SANTÉ (OMS) (2005), Plan d'action international sur le vieillissement : rapport sur la mise en œuvre, Cinquante-huitième Assemblée Mondiale de la Santé A58/19, http://www.who.int/gb/ebwha/pdf_files/WHA58/ A58_19-sp.pdf (acedido a 22-6-2007).

PAÚL, C. e A. M. Fonseca, et al (2005) “Satisfação e Qualidade de Vida em Idosos Portugueses”, in C. Paul e A. M. Fonseca (coords), Envelhecer em Portugal, Lisboa, Climepsi Editores, pp. 76-95.

PORTUGAL (2006), Programa Nacional para a Saúde das Pessoas Idosas, Lisboa, Ministério da Saúde, Direcção-Geral da Saúde.

PORTUGAL, (2004), Plano Nacional Saúde 2004-2010 : mais saúde para todos, Lisboa, Ministério da Saúde, Direcção-Geral da Saúde, http://www. dgsaude.minsaude.pt/pns/media/pns_vol2.pdfol. II (acedido a 1-10-2007)

VALLESPIR, J. e M. Morey (2007), “A Participação dos Idosos na Sociedade : Integração VS. Segregação”, A.R. Osório e F. C. Pinto (coords), Pessoas Idosas : Contexto Social e Intervenção Educativa, Lisboa, Instituto Piaget, pp. 225-252.

\section{RESUMOS}

Vivemos numa sociedade envelhecida, em que o número de idosos aumenta em relação aos jovens. As organizações de saúde procuram encontrar respostas que desafiem os cenários mais pessimistas. É importante que os idosos se mantenham activos, participantes e integrados na sociedade onde vivem. No nosso país, ainda se constata que para algumas pessoas muito idosas, viver mais tempo não é necessariamente continuar a viver com qualidade e inseridos na sua comunidade. É necessário definir políticas e estratégias globais que apliquem os princípios do envelhecimento activo, em todas as dimensões do cuidar à pessoa idosa, seja na família, seja institucionalmente. 
We live in an aged society, in which the number of old people increases in relation to the young people. The health organizations try to find answers that defy the most pessimistic scenarios. It is important that old people keep active, participant and fully integrated in the communities they live in. In our country, for some elderly to live longer does not mean necessarily to have better quality of life and to be integrated in the community. It is crucial to define policies and global strategies based on the principles of the Active Ageing that cover all the family and institutional dimensions of elderly care.

\section{ÍNDICE}

Palavras-chave: envelhecimento activo, políticas sociais e de saúde, suporte social Keywords: active ageing, society and health polices, social support 